Reprod. Nutr. Dévelop., 1985, 25 (4 A), 671-688.

\title{
Thermosensibilité de la différenciation sexuelle chez I'Amphibien Urodèle, Pleurodeles waltlii Michah. Conditions pour obtenir l'inversion du phénotype sexuel de toutes les femelles génétiques sous l'action de la température d'élevage
}

\author{
C. DOURNON, Ch. HOUILLON
}

avec la collaboration technique de Madame SZMUTYK, de Messieurs DESROSIERS et LORTHIOIT

Laboratoire de Biologie Animale, Université P.-et-M.-Curie

9, quai Saint-Bernard, 75230 Paris Cedex 05, France.

Summary. Thermosensitivity of sexual differentiation in the newt, Pleurodeles waltlii Michah. (urodele amphibian). Obtention of $100 \%$ sex reversal in genotypic females by heat treatment.

The sex reversal of genotypic females by heat treatment of the larvae at $30^{\circ} \mathrm{C}$ has been demonstrated previously in the newt, Pleurodeles waltiii (Dournon and Houillon, 1984). The aim of the present study was to define the conditions under which $100 \%$ of the genotypic females could be sex-reversed. We examined the effects on sexual differentiation of three different high temperatures and of various durations of larval exposure to these temperatures.

A total of 2304 larvae from 15 different mating pairs were divided into 29 experimental lots and 15 control lots. Control larvae were reared at ambient laboratory temperature $\left(20 \pm 2{ }^{\circ} \mathrm{C}\right)$. Experimental larvae were first reared at ambient temperature up to a given stage, then shifted to 30,31 or $32{ }^{\circ} \mathrm{C}$ for a definite period and finally returned to ambient temperature. Heat treatment started between stages 30 and 50 and was stopped between stage 45 and 2 months after metamorphosis. The percentage of sex-reversed females was expressed as an inversion coefficient calculated from the sex ratio in the experimental lots and the corresponding control lots.

The thermosensitivity of sexual differentiation of the gonads differed between individuals but did not differ significantly between the mating pairs studied. Moreover, heat treatment was more efficient in reversing the female phenotype at 31 and $32{ }^{\circ} \mathrm{C}$ than at $30^{\circ} \mathrm{C}$ and depended on the stages at which the larvae were exposed. To obtain sex reversal at $30^{\circ} \mathrm{C}$ it was necessary to treat the females between stages 43 and 45 , but even then not all responded, no matter how long they were exposed to $30^{\circ} \mathrm{C}$ after stage 45 . Some sex reversal occurred at 31 and $32{ }^{\circ} \mathrm{C}$ without treatment between these stages, but treatment was required to obtain $100 \%$ reversal. Therefore, the period between stages 43 and 45 was critical for the action of temperature on the sexual differentiation of the gonads. A total of 551 genotypic $Z W$ and WW female larvae were obtained from three matings ( $Z Z$ standard males $\times W W$ thelygenous females and heat-induced $Z W$ neo-male $\times$ WW thelygenous female). Of these larvae, 254 which were reared at $32{ }^{\circ} \mathrm{C}$ between stages 43 and 56 or between stages 43 and 54 acquired a male phenotype (heat-induced ZW neomales and heat-induced WW neo-males), whereas all of the 297 control larvae reared at $20 \pm 2{ }^{\circ} \mathrm{C}$ were phenotypic females. The minimal period of exposure to $32{ }^{\circ} \mathrm{C}$, needed for producing $100 \%$ of sex-reversed females, extended from stage 43 to stage 54 . 
At stage 43 , the genital ridges began to swell. At stage 54 the first indications of sexual differentiation of the gonads appeared. Therefore, the period sensitive to treatment at $32{ }^{\circ} \mathrm{C}$ was before morphological differentiation of the gonads.

\section{Introduction.}

L'inversion du phénotype femelle obtenue chez Pleurodeles waltlii à la suite de l'élevage des larves à une température supérieure à la normale (Houillon et Dournon, 1978) a été amplement prouvée par l'analyse génétique de leur descendance. $L^{\prime}$ expérience initiale reposait sur l'élevage pendant plusieurs mois à la température de $30^{\circ} \mathrm{C}$, de jeunes larves dont le génotype femelle $\mathrm{ZW}$ était connu. A la suite de ce traitement, certains individus présentaient tous les caractères externes de la maturité sexuelle mâle (thermo-néo-mâles ZW). Croisés avec des femelles standard ZW, les thermo-néo-mâles $Z W$ avaient des descendances dont la sex-ratio était conforme aux prévisions théoriques : $25 \%$ de mâles et $75 \%$ de femelles. Parmi les femelles issues de ces croisements, il a pu être reconnu des animaux qui possédaient le génotype femelle WW (femelles thélygènes). Les descendances issues des croisements entre mâles standard $Z Z$ et femelles WW ou entre thermo-néo-mâles ZW et femelles WW étaient constituées de $100 \%$ de femelles phénotypiques (Dournon et Houillon, 1984).

A partir de l'élevage à $30^{\circ} \mathrm{C}$, l'inversion du phénotype femelle n'a pas été obtenue dans tous les cas ; certaines larves ont évolué en femelles phénotypiques conformément à leur génotype tandis que d'autres sont devenues intersexuées. De plus, ce traitement thermique a été pratiqué pendant une longue période (4 mois) ; il débutait à l'éclosion et se terminait 2 mois après la métamorphose. II convenait d'améliorer l'efficacité du traitement de façon à obtenir l'inversion complète du phénotype sexuel de toutes les femelles génétiques tout en cherchant à réduire à son minimum la période d'exposition à la température élevée.

A cet effet, de nombreux protocoles ont été établis à partir de descendances standard et les élevages réalisés en modifiant trois paramètres qui interviennent dans un traitement thermique : le stade initial, le stade final et la température ellemême. Les résultats obtenus indiquent que chez Pleurodeles walt/ii la sensibilité de la différenciation sexuelle à l'action de la température varie selon les individus, mais ne présente aucune différence significative selon les descendances étudiées ; de plus, l'efficacité du traitement varie selon les stades d'exposition des larves et augmente en fonction de la température. Des protocoles établis à partir de descendances de femelles thélygènes WW ont permis de définir avec précision des conditions de température anormalement élevée et des périodes de traitement entraînant inéluctablement l'inversion du phénotype sexuel de toutes les femelles génétiques.

\section{Matériel et méthodes.}

Elevage et traitements thermiques. - Les conditions normales d'élevage et de reproduction au laboratoire de Pleurodeles waltlii ont été définies par Gallien 
(1952). La table chronologique du développement établie à $18^{\circ} \mathrm{C}$ (Gallien et Durocher, 1957) est utilisable à la température ambiante de $20 \pm 2{ }^{\circ} \mathrm{C}$ après une légère correction de l'âge des animaux. Mais aux températures élevées de 30 et $32{ }^{\circ} \mathrm{C}$, le développement larvaire est profondément modifié. Les modifications les plus évidentes concernent la vitesse du développement et la taille des larves. $A 30^{\circ} \mathrm{C}$, la température accélère le développement larvaire et provoque une réduction de la longueur totale des larves. Celles-ci se métamorphosent vers l'âge de 66 jours, soit 1 mois avant les larves témoins élevées à $20 \pm 2{ }^{\circ} \mathrm{C}$; leur longueur est alors de $4-5 \mathrm{~cm}$ tandis que celle des témoins est de $7 \mathrm{~cm}$. A $32{ }^{\circ} \mathrm{C}$, malgré une température encore plus élevée le développement n'est pas accéléré, les larves se développent à la même vitesse qu'à $20 \pm 2{ }^{\circ} \mathrm{C}$; mais à $32{ }^{\circ} \mathrm{C}$ on constate la même réduction de taille qu'à $30^{\circ} \mathrm{C}$ (Dournon, 1981). Dans le présent travail, quelle que soit la température d'élevage, la définition des stades exclut par conséquent tout critère d'âge et de longueur des larves et ne retient de la table du développement que les critères reposant sur l'apparition et l'aspect morphologique des membres antérieurs et postérieurs.

Le traitement thermique correspond à l'élevage des larves à des températures de 30,31 ou $32{ }^{\circ} \mathrm{C}$ appliquées d'une façon constante durant l'expérience ou appliquées en paliers, d'abord à 31 ou à $32^{\circ} \mathrm{C}$ jusqu'à un stade donné, puis à $30^{\circ} \mathrm{C}$ jusqu'à la métamorphose. Le traitement débute à différents stades larvaires, au plus tôt avant l'éclosion, au stade 30 , et au plus tard au stade 50 . II est interrompu au plus tôt au stade 45 et au plus tard 2 mois après la métamorphose (stade $56+2$ mois). En dehors de la période d'application de ce traitement, les animaux sont élevés dans les conditions de température ambiante du laboratoire à $20 \pm 2{ }^{\circ} \mathrm{C}$.

L'étude a porté sur un total de 2304 Pleurodèles ayant au moins atteint l'âge de 3 mois. Ces animaux proviennent de 15 descendances différentes. Chacune d'entre elles est à la base d'une série expérimentale qui comporte toujours un lot d'animaux témoins élevés à la température ambiante et un ou plusieurs lots d'animaux expérimentaux soumis au traitement thermique. Pour chaque lot d'animaux, la sex-ratio a été établie à partir du diagnostic du phénotype sexuel des gonades (Dournon et Houillon, 1984).

\section{TABLEAU 1}

Pourcentages théoriques de máles et de femelles phénotypiques issus de croisements entre individus standard et individus expérimentaux.

(élevage à la température ambiante $20 \pm 2{ }^{\circ} \mathrm{C}$ )

\begin{tabular}{|c|c|c|c|}
\hline & \multirow{2}{*}{ Parents } & \multicolumn{2}{|c|}{$\begin{array}{c}\text { Phénotype } \\
\text { de la descendance }\end{array}$} \\
\hline & & $\% \quad{ }^{*}$ & $\% q$ \\
\hline$O^{*}$ standard ZZ & $\times$ 甲 standard ZW & 50 & 50 \\
\hline $\mathrm{O}^{*}$ standard ZZ & $\times \subsetneq$ thélygène WW & 0 & 100 \\
\hline Thermo-néo- $\sigma \mathrm{ZW}$ & $\times$ standard ZW & 25 & 75 \\
\hline Thermo-néo- $\sigma \mathrm{ZW}$ & $\times$ O thélygène WW & 0 & 100 \\
\hline
\end{tabular}


Descendances utilisées. - En plus des Pleurodèles standard, mâles ZZ et femelles $Z W$, la disponibilité de thermo-néo-mâles $Z W$ et de femelles thélygènes WW autorise des croisements dont la descendance aura une sex-ratio prévisible (tabl. 1). Pour les descendances présentant des mâles et des femelles phénotypiques, élevés à la température ambiante, la sex-ratio observée est toujours remarquablement proche de la sex-ratio théorique (Dournon et Houillon, 1984). Cette conformité entre la prévision théorique et les résultats obtenus à la température ambiante donnera toute leur signification aux déviations de la sex-ratio observées à la suite de l'élevage à $30-32^{\circ} \mathrm{C}$. Les résultats seront encore plus démonstratifs lorsque s'agissant de descendances constituées uniquement d'animaux à génotype femelle, on observera des animaux à phénotype mâle après l'élevage à $30-32{ }^{\circ} \mathrm{C}$.

Coefficient d'inversion du phénotype femelle. - La comparaison des résultats entre les différents lots expérimentaux est basée sur un coefficient d'inversion (C.I.) dont la valeur traduit la plus ou moins grande efficacité du traitement thermique. L'établissement de ce coefficient repose sur les sex-ratio dans une même descendance, des animaux du lot témoin et de ceux d'un lot expérimental.

La déviation de la sex-ratio de chaque lot expérimental par rapport à celle du lot témoin fait l'objet d'un calcul du test d'homogénéité du $X^{2}$. Lorsque le résultat $n^{\prime}$ est pas significatif ( $\left.X^{2} \leqslant 3,841 ; d d=1 ; p=0,05\right)$ il s'agit d'un lot expérimental pour lequel on peut considérer que le traitement thermique a été sans effet décelable ; la valeur du C.I. est alors considérée comme nulle (C.I. = 0). Par contre, lorsque le résultat du test est significatif $\left(X^{2}>3,841\right)$ ou lorsqu'il y a un effet décelable de la température (présence d'intersexués ou de thermo-néo-mâles), on est en droit d'affirmer que le traitement thermique a entraîné l'inversion du phénotype sexuel d'un certain nombre de femelles génétiques. Dans ces cas, le coefficient d'inversion est calculé à partir de l'expression simplifiée de la formule :

$$
\text { C.I. }=\frac{\left(e_{2}-e_{1}\right)}{e_{2}} \times 100 \text { soit C.I. }=1001-\frac{e_{1} / E}{t / T}
$$

dans laquelle, $T=$ nombre total d'animaux dans le lot témoin,

$$
\begin{aligned}
\mathrm{t}= & \text { nombre de femelles dans le lot témoin, } \\
\mathrm{E}= & \text { nombre total d'animaux dans le lot expérimental, } \\
\mathrm{e}_{1}= & \text { nombre de femelles dans le lot expérimental, } \\
\mathrm{e}_{2}= & \text { nombre théorique de femelles qui auraient été obtenues dans } \\
& \text { le lot expérimental s'il avait été élevé à la température } \\
& \text { ambiante. }
\end{aligned}
$$

$$
e_{2}=\frac{t \times E}{T} \text {. }
$$

Le C.I. rend compte du nombre d'individus qui, sur 100 femelles génétiques (ZW ou WW), se sont différenciés partiellement (intersexués) ou totalement (thermo-néo-mâles) en mâles. Plus le C.I. est élevé, plus le traitement a été efficace. Lorsque sa valeur est maximale (C.I. = 100), toutes les femelles génétiques sont inversées en mâles phénotypiques. 


\section{Résultats et interprétation.}

Les séries expérimentales, les protocoles correspondants et les résultats chiffrés pour les 44 lots d'animaux à la base du présent travail sont consignés dans les tableaux 2 et 4 . Une représentation graphique établie pour chacun de ces tableaux permet de visualiser la plus ou moins grande efficacité des traitements en fonction des stades et des températures appliquées (fig. 1 et 3 ).
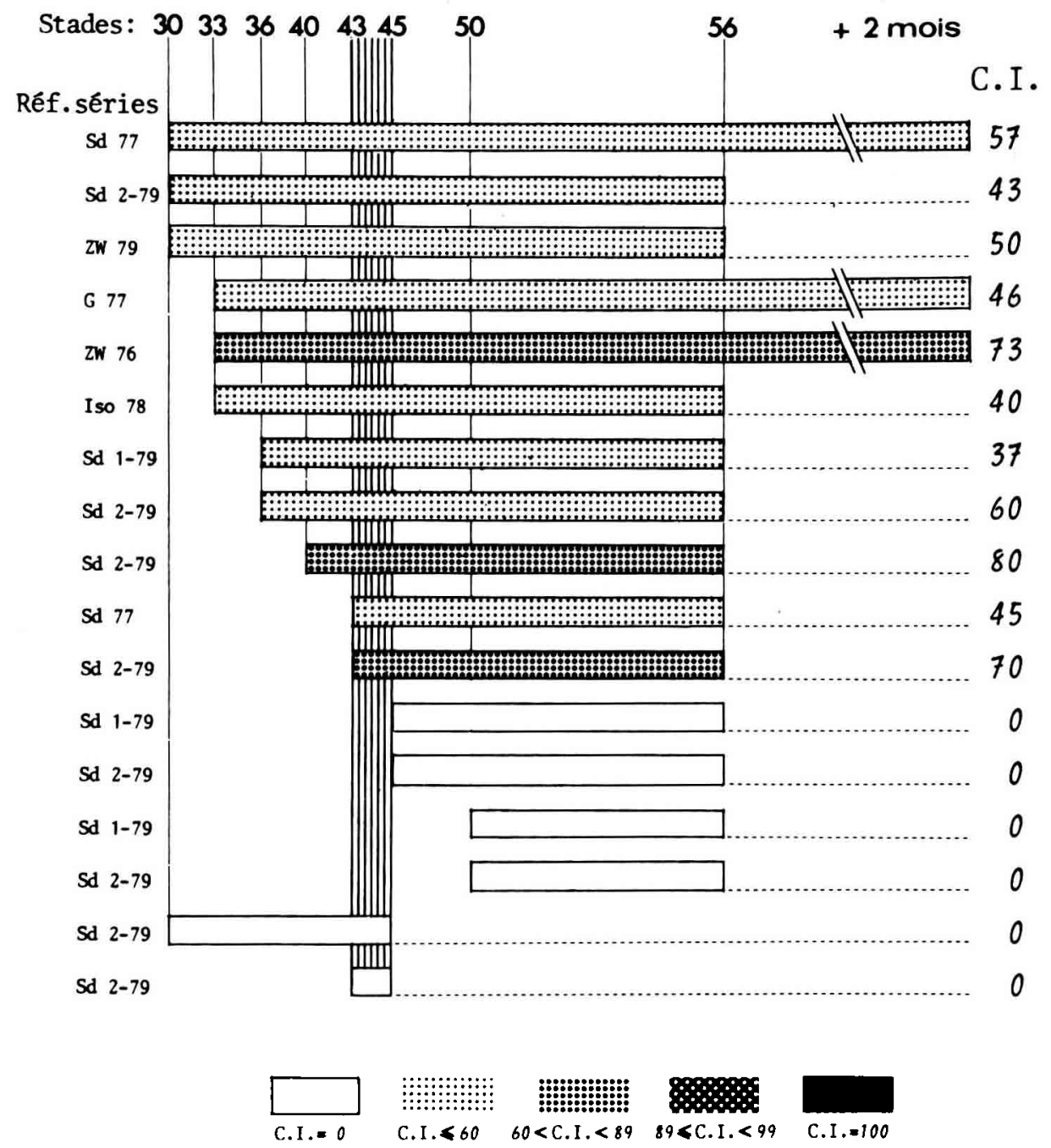

FIG. 1. - Comparaison des effets sur la sex-ratio de traitements à $30{ }^{\circ} \mathrm{C}$ appliqués aux larves de $P$. waltlii pour obtenir l'inversion du phénotype femelle (C.I. = coefficient d'inversion). Pour être efficace, le traitement doit commencer au plus tard à partir du stade 43 mais peut être interrompu à la fin de la métamorphose (st. 56). La phase larvaire comprise entre les stades 43 à 45 (hachures verticales) est une " période critique " en dehors de laquelle on n'obtient aucune inversion du phénotype femelle à $30^{\circ} \mathrm{C}$. 


\section{Inversion sexuelle à $30^{\circ} \mathrm{C}$.}

1.1. Thermosensibilité selon les individus. - Dans l'expérience initiale montrant l'inversion du phénotype sexuel femelle, il avait été obtenu 9 thermo-néomâles, 2 intersexués et 4 femelles avec des ovaires normaux (Houillon et Dournon, 1978). Une telle variabilité de réponse se retrouve dans tous les lots expérimentaux pour lesquels un traitement à $30^{\circ} \mathrm{C}$ est efficace (C.I. $>0$; tabl. 2). Bien que soumis au même traitement thermique, les animaux d'un même lot expérimental, par conséquent appartenant à la même descendance, ont une différenciation sexuelle dont la sensibilité à l'action de la température n'est pas la même. Parmi les animaux à génotype femelle certains ont évolué en mâles phénotypiques, d'autres en femelles phénotypiques, d'autres enfin sont plus ou moins inversés et présentent des gonades avec des degrés divers d'intersexualité. Si l'inversion est presque complète, il ne persiste que quelques ovocytes noyés dans les structures testiculaires; par contre, si l'inversion est à peine marquée, les gonades sont des ovaires typiques avec seulement un petit nodule testiculaire, le plus souvent situé à la partie antérieure de l'ovaire. Tous les intermédiaires sont

\section{TABLEAU 2}

Phénotypes sexuels obtenus à la suite de l'élevage des larves de $\mathrm{P}$. waltiii à la température de $30^{\circ} \mathrm{C}$. Chaquue série comporte un lot témoin et un ou plusieurs lots expérimentaux. Pour chaque lot expérimental, l'indication entre parenthèses correspond au stade du début et au stade de la fin du traitement. La figure 1 est la représentation schématique de ce tableau.

\begin{tabular}{|c|c|c|c|c|c|c|c|c|c|}
\hline \multirow{2}{*}{$\begin{array}{l}\text { Réf. } \\
\text { séries }\end{array}$} & \multirow{2}{*}{\multicolumn{2}{|c|}{ Origine }} & \multirow[b]{2}{*}{ Lots } & \multicolumn{4}{|c|}{ Nombres d'animaux } & \multirow{2}{*}{$\begin{array}{c}x^{2} \\
\text { (seuil : } \\
3,841 \text { ) }\end{array}$} & \multirow[b]{2}{*}{ C.I. } \\
\hline & & & & $\sigma^{\prime}$ & $q$ & ? & total & & \\
\hline \multirow[t]{2}{*}{$2 W 76$} & o ZZ & $\times \& W W$ & témoins & 0 & - & 10 & 10 & & \\
\hline & & & $30^{\circ} \mathrm{C}$ (st. 33 -st. $56+2$ mois) & 9 & 2 & 4 & 15 & 13,095 & 73 \\
\hline \multirow[t]{2}{*}{ G 77} & o ZW & $\times \& Z W$ & témoins & 18 & - & 40 & 58 & & \\
\hline & & & $30^{\circ} \mathrm{C}$ (st. 33-st. $56+2$ mois $)$ & 34 & - & 20 & 54 & 11,388 & 46 \\
\hline \multirow[t]{3}{*}{ Sd 77} & $\mathrm{O}^{*} \mathrm{ZZ}$ & $\times \& \mathrm{ZW}$ & témoins & 43 & - & 49 & 92 & & \\
\hline & & & $30^{\circ} \mathrm{C}$ (st. 30 -st. $56+2$ mois) & 26 & 15 & 12 & 53 & 12,945 & 57 \\
\hline & & & $30^{\circ} \mathrm{C}$ (st. $43-$ st. 56 ) & 17 & - & 7 & 24 & 4,452 & 45 \\
\hline \multirow[t]{2}{*}{ Iso 78} & transpl. & 1. nucléaire & témoins & 0 & - & 3 & 3 & & \\
\hline & $\mathrm{ZW}$ & & $30^{\circ} \mathrm{C}$ (st. 33-st. 56) & 0 & 2 & 3 & 5 & 1,600 & $40\left(^{*}\right)$ \\
\hline \multirow[t]{2}{*}{ ZW 79} & o ZZ & $\times \& W W$ & témoins & 0 & - & 4 & 4 & & \\
\hline & & & $30^{\circ} \mathrm{C}$ (st. 30-st. 56) & 3 & 1 & 4 & 8 & 3,000 & $50\left(^{*}\right)$ \\
\hline \multirow[t]{4}{*}{ Sd 1-79 } & o* ZZ & $\times \& \mathrm{ZW}$ & témoins & 23 & - & 25 & 48 & & \\
\hline & & & $30^{\circ} \mathrm{C}$ (st. 36-st. 56) & 29 & 2 & 15 & 46 & 3,685 & $37(*)$ \\
\hline & & & $30{ }^{\circ} \mathrm{C}$ (st. 45-st. 56) & 15 & - & 13 & 28 & 0,226 & 0 \\
\hline & & & $30^{\circ} \mathrm{C}$ (st. 50-st. 56) & 14 & - & 12 & 26 & 0,237 & 0 \\
\hline \multirow[t]{9}{*}{ Sd 2-79 } & or ZZ & $\times q \mathrm{ZW}$ & témoins & 39 & - & 46 & 85 & & \\
\hline & & & $30^{\circ} \mathrm{C}$ (st, 30-st. 56) & 37 & 3 & 18 & 58 & 6,282 & 43 \\
\hline & & & $30^{\circ} \mathrm{C}$ (st. 36-st. 56) & 38 & 2 & 11 & 51 & 13,936 & 60 \\
\hline & & & $30^{\circ} \mathrm{C}$ (st. 40 -st. 56) & 41 & - & 5 & 46 & 19,928 & 80 \\
\hline & & & $30^{\circ} \mathrm{C}$ (st. 43-st. 56) & 41 & - & 8 & 49 & 18,489 & 70 \\
\hline & & & $30^{\circ} \mathrm{C}$ (st. 45-st. 56) & 24 & - & 16 & 40 & 2,124 & 0 \\
\hline & & & $30^{\circ} \mathrm{C}$ (st. 50-st. 56) & 11 & - & 21 & 32 & 1,281 & 0 \\
\hline & & & $30^{\circ} \mathrm{C}$ (st. 30-st. 45) & 11 & - & 19 & 30 & 0,771 & 0 \\
\hline & & & $30^{\circ} \mathrm{C}$ (st. 43-st. 45) & 19 & - & 30 & 49 & 0,634 & 0 \\
\hline
\end{tabular}

(*) Pour ces lots, le $X^{2}$ n'étant pas significatif, le C.I. est par convention nul. Cependant, la présence de máles, à génotype femelle, et celle des individus intersexués, apparus à la suite des traitements thermiques prouvent l'efficacité de ces derniers et justifient le calcul du C.I. 
possibles entre ces deux cas extrêmes, notamment des gonades hermaphrodites dans lesquelles des spermatozoïdes mûrs voisinent avec des ovocytes en fin de vitellogenèse.

La variabilité individuelle de la réponse au traitement thermique ou thermosensibilité individuelle, est particulièrement évidente dans la série expérimentale utilisant des isojumeaux obtenus par greffes nucléaires (Aimar, Delarue et Dournon, non publié). Il est délicat d'obtenir de tels animaux en grand nombre, néanmoins la série Iso 78 (tabl. 2) comportait encore après 3 mois d'élevage, 3 femelles pour le lot témoin et 5 animaux pour le lot expérimental $\left(30^{\circ} \mathrm{C}\right)$. Parmi ces derniers, 3 présentaient des ovaires normaux mais les 2 autres avaient des gonades intersexuées. Ainsi dans un lot d'animaux pour lesquels on pouvait s'attendre à une sensibilité identique de la différenciation sexuelle compte tenu de leur origine génétique, les animaux ont répondu à des degrés divers au traitement thermique.

1.2. Thermosensibilité selon les descendances. - Les nombres de mâles et de femelles observés dans les lots d'animaux issus de descendances différentes mais soumis aux mêmes protocoles expérimentaux, même période de traitement et même température $30^{\circ} \mathrm{C}$, ne présentent aucune différence statistiquement significative (tabl. 3). Les 3 descendances pour lesquelles le test d'homogénéité a pu être calculé, présentent donc la même sensibilité à la température d'élevage $30^{\circ} \mathrm{C}$.

Ainsi, s'il existe à $30^{\circ} \mathrm{C}$ une thermosensibilité de la différenciation sexuelle propre à chaque individu, par contre il n'existe pas de thermosensibilité propre aux descendances étudiées.

TABLEAU 3

Thermosensibilité de la différenciation sexuelle selon les descendances. Pour une même période de traitement à $30^{\circ} \mathrm{C}$, les diverses séries expérimentales ne présentent pas de différence statistiquement significative. Les descendances étudiées ont la même thermosensibilité.

\begin{tabular}{|c|c|c|c|c|c|c|}
\hline \multirow{2}{*}{ Réf. séries } & Origine & \multirow{2}{*}{$\begin{array}{c}\text { Période de traitement } \\
\text { a } 30^{\circ} \mathrm{C}\end{array}$} & \multicolumn{3}{|c|}{ Nombres d'animaux } & \multirow{2}{*}{$\begin{array}{c}x^{2} \\
\text { seuil : } 3,841\end{array}$} \\
\hline & $\sigma \times 9$ & & $\varnothing$ & $q$ & Q & \\
\hline $\begin{array}{l}\text { Sd } 1-79 \\
\text { Sd } 2-79\end{array}$ & $\begin{array}{l}\mathrm{ZZ} \times \mathrm{ZW} \\
\mathrm{ZZ} \times \mathrm{ZW}\end{array}$ & st. 36 -st. 56 & $\begin{array}{l}29 \\
38\end{array}$ & $\begin{array}{l}2 \\
2\end{array}$ & $\begin{array}{l}15 \\
11\end{array}$ & 1,537 \\
\hline $\begin{array}{l}\text { Sd } 77 \\
\text { Sd 2-79 }\end{array}$ & $\begin{array}{l}\mathrm{ZZ} \times \mathrm{ZW} \\
\mathrm{ZZ} \times \mathrm{ZW}\end{array}$ & st. 43 -st. 56 & $\begin{array}{l}17 \\
41\end{array}$ & $\begin{array}{l}0 \\
0\end{array}$ & $\begin{array}{l}7 \\
8\end{array}$ & 1,681 \\
\hline $\begin{array}{l}\text { Sd } 1-79 \\
\text { Sd } 2-79\end{array}$ & $\begin{array}{l}\mathrm{ZZ} \times \mathrm{ZW} \\
\mathrm{ZZ} \times \mathrm{ZW}\end{array}$ & st. $45-$ st. 56 & $\begin{array}{l}15 \\
24\end{array}$ & $\begin{array}{l}0 \\
0\end{array}$ & $\begin{array}{l}13 \\
16\end{array}$ & 0,301 \\
\hline $\begin{array}{l}\text { Sd 1-79 } \\
\text { Sd 2-79 }\end{array}$ & $\begin{array}{l}\mathrm{ZZ} \times \mathrm{ZW} \\
\mathrm{ZZ} \times \mathrm{ZW}\end{array}$ & st. 50 -st. 56 & $\begin{array}{l}14 \\
11\end{array}$ & $\begin{array}{l}0 \\
0\end{array}$ & $\begin{array}{l}12 \\
21\end{array}$ & 2,229 \\
\hline
\end{tabular}

1.3. Réponses au traitement à $30^{\circ} \mathrm{C}$ selon la période d'exposition. - Dans les premières séries expérimentales (ZW 76, G 77 et Sd 77 ; tabl. 2), l'élevage à $30^{\circ} \mathrm{C}$ était poursuivi 2 mois après la métamorphose. Mais lorsqu'il a été reconnu que les traitements interrompus au moment de la métamorphose étaient tout aussi efficaces, aucun élevage à cette température n'a été poursuivi au-delà (fig. 1). Cependant il convenait de rechercher les limites entre lesquelles se situait 
la période larvaire de différenciation sexuelle sensible à l'action de la température de $30^{\circ} \mathrm{C}$. L'étude a porté principalement sur une descendance, la série Sd 2-79 (tabl. 2 ; fig. 2).

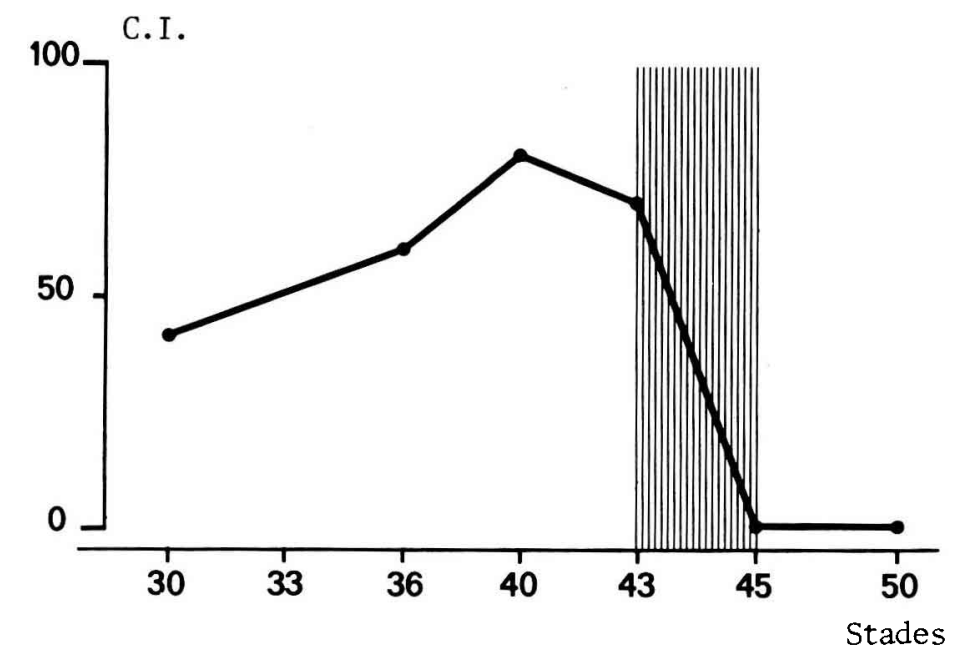

FIG. 2. - Variation pour une mềme descendance (Sd 2-79) des valeurs du C.I. en fonction du stade initial du traitement $30{ }^{\circ} \mathrm{C}$. Les traitements commencent aux stades indiqués et s'arrêtent à la métamorphose (st. 56).

Le traitement à $30^{\circ} \mathrm{C}$ est efficace $(37<\mathrm{C} .1 .<80)$ chaque fois qu'il débute avant ou au plus tard, à partir du stade 43 et qu'il se poursuit jusqu'à la métamorphose (stade 56). Par contre, le traitement est inefficace (C.I. = 0) quand il débute au stade 45 ou au stade 50 et qu'il se termine à la métamorphose. La même inefficacité se retrouve pour les lots de la série Sd 1-79 auxquels fut appliqué le même protocole. L'inefficacité du traitement s'observe également quand il débute au stade 30 ou au stade $\mathbf{4 3}$ mais qu'il est interrompu dès le stade $\mathbf{4 5}$ (fig. 1).

Les réponses des larves au traitement à $30^{\circ} \mathrm{C}$ appliqué à des stades différents du développement montrent l'importance de la période comprise entre les stades 43 et 45 . II s'agit d'une période critique qui doit être nécessairement incluse dans la durée du traitement pour que celui-ci soit efficace. Mais si elle est nécessaire, cette période critique n'est pas suffisante à elle seule puisqu'il est indispensable de poursuivre le traitement jusqu'à la métamorphose pour qu'il soit efficace. De la sorte, la période larvaire au cours de laquelle la différenciation sexuelle est sensible à la température de $30^{\circ} \mathrm{C}$ est comprise entre le stade 43 et la métamorphose. Cependant, l'inversion du phénotype femelle n'étant jamais obtenue pour toutes les femelles génétiques, l'efficacité du traitement thermique à $30^{\circ} \mathrm{C}$ n'est jamais totale.

2. Inversion sexuelle à 31 et à $32{ }^{\circ} \mathrm{C}$. Les protocoles précédents avaient en commun la température d'élevage a $30^{\circ} \mathrm{C}$, tandis que la période d'exposition et l'origine des animaux variaient. Dans les protocoles suivants, la température est modi- 
fiée tandis que la période d'exposition et l'origine des animaux varient comme à $30{ }^{\circ} \mathrm{C}$. La température a été soit maintenue constante à 31 ou à $32{ }^{\circ} \mathrm{C}$, soit modifiée au cours du traitement passant à un stade donné de 31 ou $32^{\circ} \mathrm{C}$ à $30^{\circ} \mathrm{C}$ (tabl. 4 ; fig. 3 ).

\section{TABLEAU 4}

Phénotypes sexuels obtenus à la suite de l'élevage des larves de P. waltlii à la température de 31 ou $32{ }^{\circ} \mathrm{C}$. Chaque série comporte un lot témoin et un ou plusieurs lots expérimentaux. L'indication entre parenthèses précise pour chaque lot expérimental la température appliquée d'un stade à l'autre. La figure 3 est la représentation schématique de ce tableau.

\begin{tabular}{|c|c|c|c|c|c|c|c|c|c|}
\hline \multirow{2}{*}{$\begin{array}{c}\text { Réf. } \\
\text { séries }\end{array}$} & \multirow{2}{*}{\multicolumn{2}{|c|}{ Origine }} & \multirow[b]{2}{*}{ Lots } & \multicolumn{4}{|c|}{ Nombres d'animaux } & \multirow{2}{*}{$\begin{array}{c}x^{2} \\
- \text { (seuil : } \\
3,841 \text { ) }\end{array}$} & \multirow[b]{2}{*}{ C.I. } \\
\hline & & & & $0^{*}$ & $q^{*}$ & q & total & & \\
\hline \multirow[t]{2}{*}{ H 79} & $\mathrm{O}^{*} \mathrm{ZW}$ & $x \odot \mathrm{ZW}$ & témoins & 38 & - & 88 & 126 & & \\
\hline & & & $\begin{array}{l}30^{\circ} \mathrm{C} \text { (st. } 40 \text {-st. 50) puis } \\
30^{\circ} \mathrm{C} \text { (st. } 50 \text {-st. 56) }\end{array}$ & 95 & 3 & 5 & 103 & 99,081 & 93 \\
\hline \multirow[t]{2}{*}{ Sd 2-79 } & $\sigma \mathbf{Z Z}$ & $\times \subsetneq \mathrm{ZW}$ & témoins & 39 & - & 46 & 85 & & \\
\hline & & & $\begin{array}{l}32^{\circ} \mathrm{C} \text { (st. 43-st. 53) puis } \\
30^{\circ} \mathrm{C} \text { (st. 53-st. 56) }\end{array}$ & 43 & - & 1 & 44 & 33,523 & 96 \\
\hline \multirow[t]{2}{*}{$\alpha 80$} & o ZW & $\times \& W W$ & témoins & 0 & - & 57 & 57 & & \\
\hline & & & $\begin{array}{l}32{ }^{\circ} \mathrm{C} \text { (st. } 40 \text {-st. 47) puis } \\
30^{\circ} \mathrm{C} \text { (st. 47-st. 56) }\end{array}$ & 23 & 1 & 12 & 36 & 50,137 & 67 \\
\hline \multirow[t]{2}{*}{ Sd 1-80 } & $\sigma^{*} \mathbf{Z Z}$ & $\times \odot \mathrm{ZW}$ & $\begin{array}{l}\text { témoins } \\
32^{\circ} \mathrm{C} \text { (st. } 45 \text {-st. 56) } \\
32^{\circ} \mathrm{C} \text { (st. 50-st. 56) } \\
32^{\circ} \mathrm{C} \text { (st. } 40 \text {-st. 50) }\end{array}$ & $\begin{array}{l}61 \\
39 \\
36 \\
19\end{array}$ & $\begin{array}{l}- \\
- \\
-\end{array}$ & $\begin{array}{r}60 \\
2 \\
14 \\
16\end{array}$ & $\begin{array}{r}121 \\
41 \\
50 \\
35\end{array}$ & $\begin{array}{r}19,680 \\
6,652 \\
0,178\end{array}$ & $\begin{array}{r}90 \\
44 \\
0\end{array}$ \\
\hline & & & $\begin{array}{l}32{ }^{\circ} \mathrm{C} \text { (st. } 40 \text {-st. 50) puis } \\
30^{\circ} \mathrm{C} \text { (st. 50-st. 56) }\end{array}$ & 35 & - & 2 & 37 & 24,118 & 89 \\
\hline Sd-Toul 81 & $\sigma Z Z$ & $\times \propto Z W$ & $\begin{array}{l}\text { témoins } \\
32{ }^{\circ} \mathrm{C} \text { (st. } 50 \text {-st. } 56 \text { ) }\end{array}$ & $\begin{array}{l}13 \\
24\end{array}$ & - & $\begin{array}{l}16 \\
10\end{array}$ & $\begin{array}{l}29 \\
34\end{array}$ & 4,217 & 47 \\
\hline ZW 82 & or ZZ & $\times \& W W$ & témoins & 0 & - & 130 & 130 & & \\
\hline$\beta 83$ & o $\mathrm{ZW}$ & $\times \propto W W$ & $\begin{array}{l}31^{\circ} \mathrm{C} \text { (st. } 43 \text {-st. } 56 \text { ) } \\
\text { témoins }\end{array}$ & $\begin{array}{r}4 b \\
0\end{array}$ & $\begin{array}{l}- \\
-\end{array}$ & $\begin{array}{r}0 \\
144\end{array}$ & $\begin{array}{r}45 \\
144\end{array}$ & 174,517 & 100 \\
\hline Sd 83 & o ZZ & $\times \subsetneq \mathrm{ZW}$ & $\begin{array}{l}32^{\circ} \mathrm{C} \text { (st. 43-st. 56) } \\
\text { témoins } \\
31^{\circ} \mathrm{C} \text { (st. 50-st. 56) }\end{array}$ & $\begin{array}{r}184 \\
39 \\
30\end{array}$ & - & $\begin{array}{r}0 \\
43 \\
13\end{array}$ & $\begin{array}{r}184 \\
82 \\
44\end{array}$ & 328,163 & 100 \\
\hline ZW 84 & $\sigma^{*} \mathbf{Z Z}$ & $x \propto W W$ & $\begin{array}{l}\text { témoins } \\
32^{\circ} \mathrm{C} \text { (st. 43-st. 54) }\end{array}$ & $\begin{array}{r}0 \\
25\end{array}$ & - & $\begin{array}{r}23 \\
0\end{array}$ & $\begin{array}{l}23 \\
25\end{array}$ & 48,168 & 100 \\
\hline
\end{tabular}

2.1. Réponses aux traitements à 31 ou $32{ }^{\circ} \mathrm{C}$ selon le début de la période d'exposition. - A 31 et à $32{ }^{\circ} \mathrm{C}$, le traitement thermique appliqué jusqu'à la métamorphose est efficace même s'il commence après le stade 43 . II est d'autant plus efficace qu'il est appliqué plus tôt : quand il commence au stade 50 , l'inversion sexuelle s'avère encore possible aussi bien à $31^{\circ} \mathrm{C}$ (C.I. = 44; fig. 3) qu'à $32{ }^{\circ} \mathrm{C}$ - $($ C.I. = 44 ou 47); quand le traitement débute plus tôt, au stade 45 , il devient à $32{ }^{\circ} \mathrm{C}$ particulièrement efficace $(\mathrm{C} . \mathrm{I} .=90)$ et lorsqu'il débute au stade 43 à $32{ }^{\circ} \mathrm{C}$, ou au stade 40 à $31^{\circ} \mathrm{C}$, il est totalement efficace (C.I. $=100$ ). Grâce à de tels traitements, l'inversion du phénotype femelle se réalise dans $100 \%$ des cas (tabl. 4 ; fig. 3).

Ce dernier résultat très démonstratif fut obtenu dans deux séries expérimentales différentes. La première, ZW 82 (tabl. 4), porte sur 175 animaux issus d'une descendance monogénique femelle $Z W$ provenant du croisement entre un mâle 

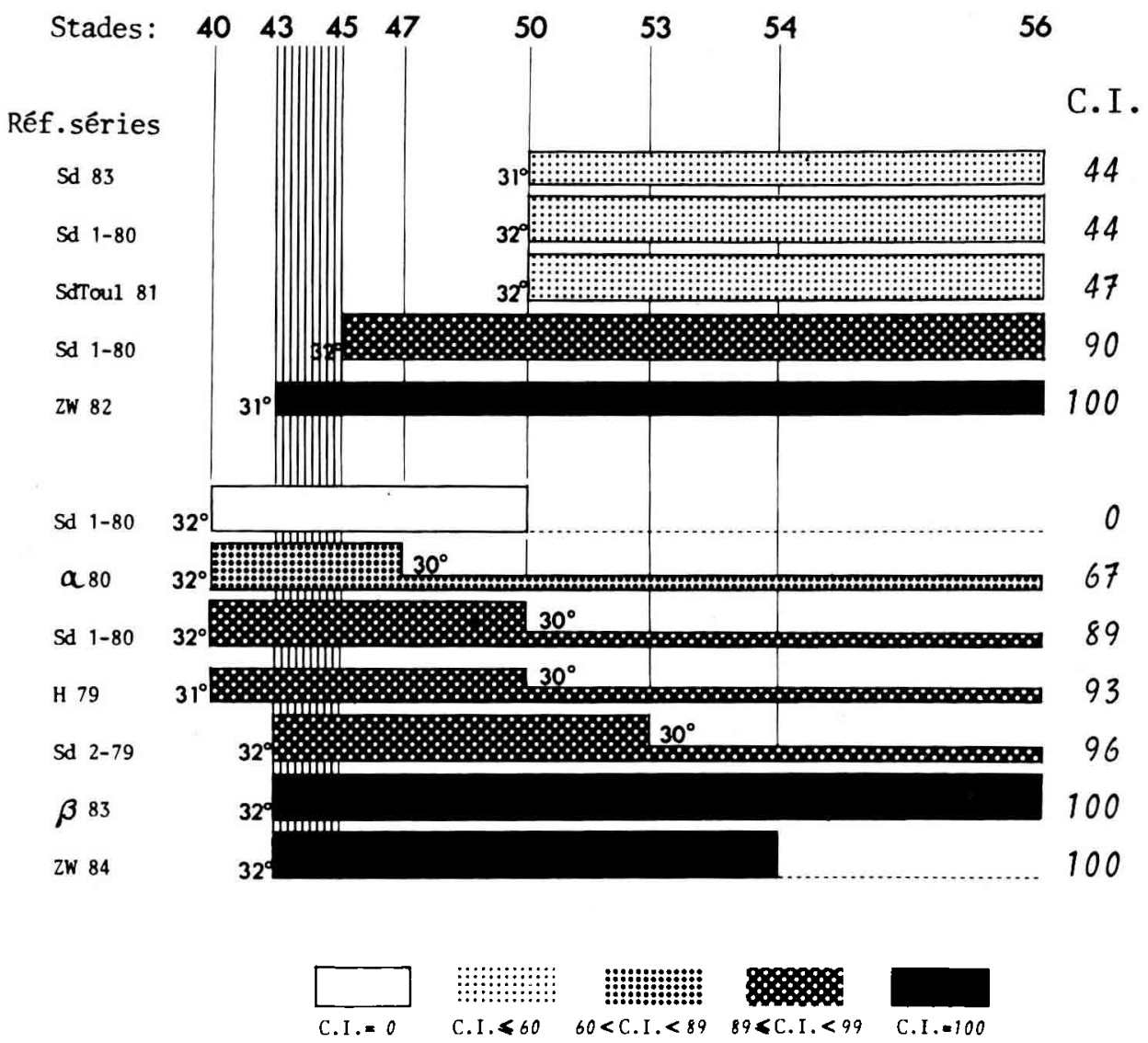

FIG. 3. - Comparaison des effets sur la sex-ratio de traitements à 31 et à $32{ }^{\circ} \mathrm{C}$ appliqués aux larves de $P$. waltlii pour obtenir 'inversion du phénotype sexuel des femelles. A $32{ }^{\circ} \mathrm{C}$, pour avoir une inversion de tous les individus $(\mathrm{C} . \mathrm{I} .=100)$, le traitement doit commencer au plus tard au stade $\mathbf{4 3}$ mais peut être interrompu dès le stade 54. La phase larvaire comprise entre les stades 43 à 45 (hachures verticales) est une "période critique " absolument nécessaire pour obtenir l'inversion dans $100 \%$ des cas.

standard ZZ et une femelle thélygene WW. Le lot témoin élevé à la température ambiante comprend 130 femelles phénotypiques; le lot expérimental élevé à $31^{\circ} \mathrm{C}$ depuis le stade 43 jusqu'à la métamorphose comprend 45 animaux tous à phénotype mâle (thermo-néo-mâles $Z W$ ). La seconde série expérimentale, $\beta 83$, porte sur 328 animaux issus d'un thermo-néo-mâle $Z W$ et d'une femelle thélygène WW. Le lot témoin élevé à la température ambiante comprend 144 femelles phénotypiques (génotype $Z W$ ou $W W$ ) ; le lot expérimental élevé à $32^{\circ} \mathrm{C}$ du stade 43 à la métamorphose comprend 184 animaux tous à phénotype mâle (thermo-néomâles ZW ou thermo-néo-mâles WW). Ainsi sur un total de 229 larves à génotype femelle, ZW ou WW, l'élevage à la température de 31 ou de $32^{\circ} \mathrm{C}$ depuis le stade 43 jusqu'à la métamorphose a entraîné la différenciation sexuelle uniquement dans le sens testiculaire. L'inversion est complète pour tous les individus, on ne rencontre aucun animal intersexué. 
Les différences entre les nombres de mâles et de femelles observés dans les lots traités à 31 ou $32{ }^{\circ} \mathrm{C}$ pendant une même période et les nombres de mâles et de femelles observés dans les lots traités à $30^{\circ} \mathrm{C}$ pendant la même période sont significatives ( $X^{2}$ inter températures ; tabl. 5) ; l'efficacité des traitements est améliorée par l'élévation de la température de 30 à $31-32{ }^{\circ} \mathrm{C}$. A ces températures, on ne rencontre qu'exceptionnellement des individus intersexués, la variabilité de la thermosensibilité qui se manifestait à $30^{\circ} \mathrm{C}$ selon les individus semble être atténuée. D'autre part, les nombres de mâles et de femelles observés dans les lots d'origine différente mais ayant subi le même traitement ne sont pas significativement différents ( $X^{2}$ intra température), la thermosensibilité est donc, comme à $30{ }^{\circ} \mathrm{C}$, la même pour toutes les descendances étudiées (tabl. 5). A $31-32{ }^{\circ} \mathrm{C}$, la différence de réponse intervient en fonction du début de la période d'exposition (fig. 3).

A $32{ }^{\circ} \mathrm{C}$, la notion de période critique définie à $30^{\circ} \mathrm{C}$ entre les stades 43 et 45 a une tout autre importance. Cette période n'est plus nécessaire à $32{ }^{\circ} \mathrm{C}$ pour que le traitement soit efficace alors qu'elle l'était à $30^{\circ} \mathrm{C}(37<\mathrm{C} . \mathrm{l}$ < $<80)$, par contre elle devient indispensable pour obtenir une totale efficacité $(C . I .=100)$.

2.2. Réponses aux traitements à 31 ou $32{ }^{\circ} \mathrm{C}$ selon la fin de la période d'exposition. - A $32{ }^{\circ} \mathrm{C}$ contrairement à $30^{\circ} \mathrm{C}$, quand le traitement débute après le stade 43 , il est encore efficace, et quand toujours à $32{ }^{\circ} \mathrm{C}$ il commence au stade 43 , il est totalement efficace. Qu'en est-il des traitements à 31 ou à $32{ }^{\circ} \mathrm{C}$ qui, débutant avant le stade 43 , sont interrompus ou modifiés avant la métamorphose?

Un traitement à $32{ }^{\circ} \mathrm{C}$ qui débute au stade 40 pour être interrompu au stade 50 (Sd $1-80$; tabl. 4) est totalement inefficace, C.I. $=0$. Or, un traitement qui débute lui aussi au stade 40 mais qui est modifié par une baisse de température de 32 à $30^{\circ} \mathrm{C}$ dès le stade 47 présente une certaine efficacité. Ce résultat obtenu à partir d'une descendance constituée à $100 \%$ de femelles génétiques $(\alpha 80$, tabl. 4) est significatif car, après traitement, $67 \%$ des individus ont des testicules $\left(X^{2}=50,1\right)$. L'efficacité du traitement augmente lorsque la modification de la température est de plus en plus tardive. Ainsi lorsque le changement a lieu au stade 50 (Sd 1-80) l'efficacité est importante, C.I. = 89. Et si le traitement débute un peu plus tard au stade 43, mais est modifié au stade 53 (Sd 2-79), il devient $d^{\prime}$ 'une efficacité presque totale, C.I. $=96$. A noter que pour ces deux traitements particulièrement efficaces les différences entre les nombres de mâles et de femelles ne sont pas significatives $\left(X^{2}=0,501\right)$. Mais ce n'est qu'avec une température constante de 31 ou $32{ }^{\circ} \mathrm{C}$ appliquée du stade 43 à la métamorphose (ZW 82 et $\beta$ 83) que le traitement est d'une efficacité totale, C.I. = 100 (fig. 3). Dans ces deux séries, les résultats sont hautement significatifs puisqu'ils ont été obtenus à partir de descendances issues de femelles thélygènes $W W\left(X^{2}=174,5\right.$ et $X^{2}=328,11$.

La période larvaire comprise entre le stade 43 et le stade 56 au cours de laquelle l'élevage à 31 ou à $32^{\circ} \mathrm{C}$ entraîne l'inversion complète du phénotype femelle dans $100 \%$ des cas peut être réduite sans pour autant altérer la totale efficacité du traitement. Ainsi, à partir de 25 larves provenant d'une descendance monogénique femelle ZW (ZW 84 ; tabl. 4) élevées du stade 43 au stade 54 à 


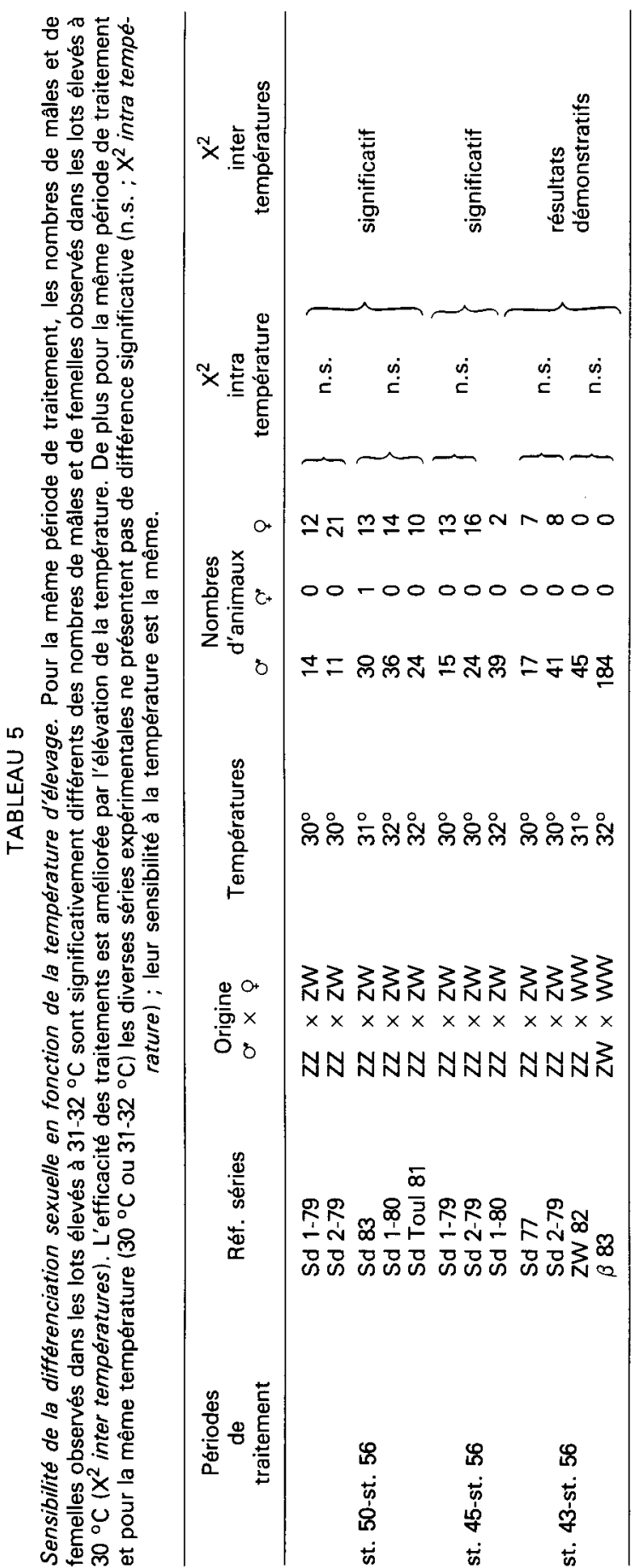


$32{ }^{\circ} \mathrm{C}$, il a été obtenu 25 mâles phénotypiques. A noter qu'il s'agit là du troisième lot expérimental issu d'une femelle thélygène $W W$, pour lequel l'élevage à 31 ou à $32{ }^{\circ} \mathrm{C}$ a été totalement efficace (fig. 3). II ne semble pas possible de réduire plus encore la période en cause en gardant néanmoins une totale efficacité, car lorsque l'élevage à $32{ }^{\circ} \mathrm{C}$ débute au stade 43 puis est ramené à $30^{\circ} \mathrm{C}$ au stade 53 jusqu'à la métamorphose, le traitement $n^{\prime}$ 'est plus totalement efficace (fig. 3 ).

On peut donc considérer que la période larvaire comprise entre les stades 43 et 54 est la période thermosensible de la différenciation sexuelle, nécessaire et suffisante, pour obtenir l'inversion du phénotype femelle à la température de $32{ }^{\circ} \mathrm{C}$ dans $100 \%$ des cas.

\section{Discussion.}

L'objectif de ce travail était de rechercher les conditions qui permettent d'obtenir l'inversion du phénotype sexuel de toutes les femelles chez Pleurodeles waltiii, sous l'action d'une température d'élevage supérieure à la normale. La démarche expérimentale qui consistait à faire varier la période de traitement et les conditions de température a montré que l'inversion complète de toutes les femelles génétiques est obtenue en élevant les larves à $32{ }^{\circ} \mathrm{C}$ entre les stades 43 et 54 . Cette période correspond à l'organogenèse gonadique depuis la mise en place des crêtes génitales jusqu'au début de la différenciation sexuelle histologique des gonades. Au stade 43, l'ébauche de la gonade n'est encore qu'une simple crête située au voisinage immédiat du blastème mésonéphrétique et dans laquelle sont localisées les cellules germinales primordiales. Aux stades 44 et 45 , l'organogenèse se poursuit par la mise en place des éléments somatiques médullaires; la gonade sexuellement indifférenciée se constitue. A ce moment du développement, toute agénésie mésonéphrétique entraîne une agénésie de la partie médullaire des gonades se traduisant par la dégénérescence des cellules germinales (Houillon, 1956). Nous montrons que cette période du développement larvaire, comprise entre les stades 43 et 45 , est critique pour obtenir l'inversion sexuelle des femelles génétiques avec une relative efficacité à $30{ }^{\circ} \mathrm{C}(70 \%$ d'inversion entre les stades 43 et 56$)$ et avec une totale efficacité à 31 ou à $32{ }^{\circ} \mathrm{C}(100 \%$ d'inversion entre les mêmes stades). Au cours de cette période se déroulent des phénomènes essentiels pour la différenciation des gonades, et problablement l'apparition du ou des facteurs thermosensibles dont la modification déclenche celle de la différenciation sexuelle, voire l'apparition des substances qui en sont responsables dans les conditions normales. Les facteurs thermosensibles semblent donc être présents très tôt, dès l'édification des crêtes génitales mais ce n'est que relativement tard que les effets de la température se traduiraient au niveau de la différenciation histologique du sexe des gonades (st. 53).

L'action d'une température de $32^{\circ} \mathrm{C}$ durant la phase de thermosensibilité de la différenciation sexuelle de la gonade se traduit par la différenciation d'un seul sexe phénotypique : tout comme les mâles génétiques $Z Z$, les femelles génétiques ZW et WW évoluent en mâles phénotypiques. Chez les larves génétiquement males qui ne sont pas affectées par l'action de la température, les phénomènes 
responsables de la différenciation testiculaire sont probablement les mêmes à la température ambiante et à $32{ }^{\circ} \mathrm{C}$. Par contre, la différenciation des gonades des larves génétiquement femelles est perturbée d'une façon telle qu'au lieu d'une différenciation ovarienne, il s'effectue une différenciation testiculaire d'apparence normale. En effet, les thermo-néo-mâles, fonctionnels, ont une physiologie sexuelle semblable à celle des mâles standard. Tout semble se passer comme si les substances responsables de la différenciation ovarienne à la température ambiante étaient à $32{ }^{\circ} \mathrm{C}$, dominées par celles de la différenciation mâle, ou encore comme si elles étaient à $32{ }^{\circ} \mathrm{C}$ inexistantes ou rendues inopérantes, permettant ainsi aux substances responsables de la masculinisation de s'exprimer.

Le mécanisme de l'action de la température sur la différenciation sexuelle des gonades est encore inconnu. Cependant nos résultats permettent d'envisager que chez Pleurodeles walt/ii, la différenciation de l'un ou l'autre sexe gonadique chez les larves à génotype femelle, dépend d'un seuil de substances. Ce seuil pourrait correspondre à une quantité critique de substances qui progressivement interviendraient (substances masculinisantes), ou n'interviendraient plus (substances féminisantes), au cours du développement larvaire et conduiraient à la différenciation testiculaire. Si le seuil n'est pas atteint au cours du traitement thermique, les gonades se différencient dans le sens ovarien conformément au génotype. Par contre, lorsque ce seuil est atteint, le traitement est efficace, la différenciation s'effectue dans le sens mâle. Bien qu'encore hypothétique, ce point de vue permet de rendre compte de la variabilité des réponses aux traitements thermiques.

La variabilité de la thermosensibilité selon les individus montre clairement que ce seuil n'est pas le même pour toutes les femelles génétiques. Elle montre aussi que le traitement sera efficace sur une larve si le seuil est atteint avant la fin du traitement et qu'il sera efficace sur toutes les larves s'il leur permet à toutes de franchir leur propre seuil critique. Cependant la conséquence de l'élévation de la température de 30 à $32{ }^{\circ} \mathrm{C}$, à savoir l'amélioration de l'efficacité des traitements, permet de penser que la quantité critique de substances responsables de la différenciation sexuelle serait elle-même atteinte sous l'influence d'un seuil de température. En effet, nos résultats montrent que la modification de la différenciation sexuelle dépend non seulement de la période d'exposition à une température donnée, c'est-à-dire de la durée d'exposition à cette température pendant la période thermosensible, mais aussi de l'élévation de la température (tabl. 5).

Ainsi a $30^{\circ} \mathrm{C}$, le traitement commencé au stade 43 doit être poursuivi jusqu'à la métamorphose pour être efficace ; commencé trop tard (st. 45) ou arrêté trop tôt (st. 45), le traitement à $30^{\circ} \mathrm{C}$ est inefficace car la quantité critique ne peut pas être atteinte. L'inefficacité d'un traitement commencé tardivement pourrait provenir d'une détermination embryonnaire qui, antérieure au début du traitement thermique, conduirait inéluctablement à la différenciation ovarienne normale. Mais cette idée ne peut être retenue car des traitements thermiques plus tardifs qui $n^{\prime}$ entraînaient aucune inversion à $30^{\circ} \mathrm{C}$, peuvent provoquer des inversions sexuelles à 31 ou à $32{ }^{\circ} \mathrm{C}$. En admettant que les facteurs thermosensibles soient de même nature dans les divers traitements, encore faudrait-il le prouver, il est remarquable de constater que le traitement à $32^{\circ} \mathrm{C}$ est totalement efficace quand 
il débute au stade 43 et se poursuit jusqu'au stade 54 , alors qu'il est sans effet à $32{ }^{\circ} \mathrm{C}$ lorsque, même commencé tôt (st. 40), il est interrompu dès le stade 50 . Dans le premier cas, la quantité critique de substances est atteinte, dans le second cas, elle ne l'est pas. Le même type d'interprétation peut être donné aux traitements qui débutent à $32^{\circ} \mathrm{C}$ et qui se poursuivent temporairement à $30^{\circ} \mathrm{C}$. Pour les larves élevées dans ces conditions le traitement à $30^{\circ} \mathrm{C}$ ne ferait que maintenir des phénomènes commencés à $32^{\circ} \mathrm{C}$. La quantité critique pourrait alors être atteinte et entraîner l'inversion. Pour les larves ne réagissant pas alors qu'elles seraient devenues mâles si le traitement avait continué à $32^{\circ} \mathrm{C}$, la poursuite de l'élevage à $30^{\circ} \mathrm{C}$ ne permet pas d'atteindre le niveau critique, la différenciation est conforme au génotype sexuel. Le traitement à $32{ }^{\circ} \mathrm{C}$ déclenche des phénomènes qui seraient plus ou moins maintenus par le traitement à $30^{\circ} \mathrm{C}$. Cette idée est renforcée par les traitements tardifs, commencés à partir du stade 50 , toujours inefficaces à $30^{\circ} \mathrm{C}$, mais efficaces à $32{ }^{\circ} \mathrm{C}$ sur certains individus. Une élévation de seulement 1 ou $2{ }^{\circ} \mathrm{C}$ de la température amplifie des phénomènes qui doivent déjà intervenir à $30^{\circ} \mathrm{C}$. Un traitement inefficace à $30^{\circ} \mathrm{C}$ parce qu'il est trop tardif devient efficace quand il commence assez tôt, la quantité critique de substances responsables de la différenciation sexuelle pouvant alors être atteinte. A $32{ }^{\circ} \mathrm{C}$, un traitement partiellement efficace lorsqu'il est tardif devient de plus en plus efficace quand il commence de plus en plus tôt jusqu'à devenir totalement efficace sur toutes les femelles génétiques.

La notion de seuil permet aussi de rendre compte de la présence d'individus intersexués. Chez ces animaux, l'inversion complète du phénotype gonadique ne peut être effective car la quantité critique de substances serait tout juste atteinte. De plus, l'aspect des gonades de ces individus permet de penser que les glandes génitales sont régionalisées. En effet chez la plupart des animaux intersexués, un testicule s'est différencié à la partie antérieure de l'ovaire. Cette disposition anatomique facilement observable chez l'animal d'âge juvénile ou adulte (Dournon et Houillon, 1984) pourrait être la conséquence de la différenciation cranio-caudale de la gonade, concomitante de l'édification spatio-temporelle du mésonéphros. Ainsi chez la larve, la région de la gonade la plus exposée à l'action de la température est la première au contact du blastème mésonéphrétique, à savoir la partie antérieure de la future glande génitale.

II serait intéressant de rechercher la limite inférieure de la température pour laquelle des traitements seraient encore efficaces sur certaines larves (température seuil). Mais compte tenu de la variabilité de la sensibilité qui existe déjà à $30^{\circ} \mathrm{C}$, l'étude ne pourra être envisagée que sur des descendances monogéniques femelles.

Le même intérêt concerne la limite supérieure de la température qui permettrait à un traitement appliqué pendant une période plus courte qu'à $32{ }^{\circ} \mathrm{C}$, d'avoir toujours une totale efficacité. Cependant, même si les larves de Pleurodeles walt/ii peuvent vivre quelque temps à des températures supérieures à $32{ }^{\circ} \mathrm{C}$, la durée du traitement compatible avec une bonne survie serait probablement trop brève pour être totalement efficace. II semble qu'avec la température de $32^{\circ} \mathrm{C}$, appliquée des stades 43 à 54, les conditions optimales pour obtenir l'inversion du phénotype gonadique de toutes les larves à génotype femelle aient été requises. 
Le traitement thermique ne pourrait-il pas être rapproché du heat-shock, bien que celui-ci n'aboutisse à aucune différenciation morphologique alors que le traitement thermique aboutit à une différenciation anatomique, physiologiquement fonctionnelle? Le heat-shock conduit à la mise en route d'un nouveau programme génétique. Si le heat-shock entraîne l'expression de protéines particulières, le traitement thermique entraîne l'expression d'un phénotype sexuel inverse de celui programmé après la fécondation. Le traitement thermique ne conduit-il pas lui aussi à une nouvelle programmation ?

L'interprétation biochimique des phénomènes intervenant dans l'action de la température sur la différenciation sexuelle s'avère particulièrement complexe en regard de résultats récents obtenus chez Pleurodeles poireti (Dournon et al., 1984). Pleurodeles waltlii et Pleurodeles poireti sont deux espèces très proches puisqu'elles s'hybrident et que leurs hybrides sont féconds (Lacroix, 1968). Pourtant, les larves de $P$. poireti à génotype sexuel mâle $\mathrm{ZZ}$ soumises à $31 \pm 1{ }^{\circ} \mathrm{C}$, évoluent en femelles phénotypiques fonctionnelles (thermo-néo-femelles $\mathrm{ZZ}$ ). Cependant, malgré la température d'élevage anormalement élevée, l'inversion du phénotype sexuel n'a pas encore été obtenue dans $100 \%$ des cas. Chez ces deux espèces très voisines, l'action d'une température supérieure à la normale se traduit donc par des effets opposés. Or ces deux espèces manifestent la même sensibilité à l'action des stéroïdes sexuels : chez $P$. walt/ii (Gallien, 1950) tout comme chez $P$. poireti (Lacroix, 1970), le benzoate d'œstradiol permet l'inversion du phénotype mâle et dans les deux cas des néo-femelles $Z Z$ fonctionnelles sont obtenues. Les traitements hormonaux efficaces commencent tardivement lorsque la gonade est sexuellement indifférenciée (st. 52a). Les traitements thermiques commencés à partir de ce stade seraient inefficaces car la singularité de ces traitements chez les deux espèces est qu'ils doivent débuter très tôt, lors de l'édification des crêtes génitales. Ces importantes différences chronologiques dans l'action des deux facteurs épigénétiques, la température et les hormones sexuelles, semblent indiquer une différence entre leur niveau ou leur mode d'action. Cependant, on peut envisager que la température agit au début de la stéroïdogenèse et qu'un certain délai est nécessaire pour la modifier. Les hormones sexuelles qui ont une action plus tardive, interviendraient sur les récepteurs gonadiques. II faut enfin remarquer que chez $P$. poireti, les deux facteurs épigénétiques ont une action féminisante.

Parmi les vertébrés tétrapodes, les Reptiles ont déjà fait l'objet d'études concernant la thermosensibilité de la différenciation des gonades (revues de Bull, 1980, 1983 et de Pieau, 1975, 1985). Par des modifications de la température au cours d'incubations artificielles des œufs, des périodes minimales d'exposition des œufs à diverses températures ont été définies pour obtenir $100 \%$ d'individus du même sexe. Chez les tortues (Bull et Vogt, 1981; Pieau et Dorizzi, 1981 ; Yntema, 1979 ; Yntema et Mrosovsky, 1982), les traitements thermiques peuvent commencer lorsque la gonade est sexuellement indifférenciée sur le plan histologique. Ils sont interrompus quand les premières manifestations de la différenciation des gonades sont devenues histologiquement reconnaissables. Par rapport au Pleurodèle, les traitements appliqués aux Chéloniens diffèrent par un début plus tardif en regard de l'organogenèse gonadique et aussi par les faibles 
écarts de température qui permettent d'obtenir uniquement des individus de l'un ou l'autre sexe. Ainsi à partir d'une température pivot, variant entre 27 et $31^{\circ} \mathrm{C}$ selon les espèces de tortue et pour laquelle s'effectue la différenciation des deux sexes, il suffit d'élever ou d'abaisser de 1 ou $2^{\circ} \mathrm{C}$ la température d'incubation pour obtenir soit $100 \%$ de femelles, soit $100 \%$ de mâles (Bull, 1980). Chez $P$. waltiii comme chez $P$. poireti, l'élevage à la température ambiante $\left(20 \pm 2{ }^{\circ} \mathrm{C}\right)$ aboutit toujours à une sex-ratio normale et il faut une élévation de température d'au moins $10^{\circ} \mathrm{C}$ pour obtenir $100 \%$ de femelles génétiques masculinisées chez $P$. waltlii et $61 \%$ de mâles génétiques féminisés chez $P$. poireti.

Les conditions expérimentales qui permettent d'obtenir $100 \%$ de thermonéo-mâles chez $P$. waltiii ont été mises à profit pour obtenir des thermo-néomâles WW (série $\beta 83$; tabl. 4). De tels animaux ont été effectivement reconnus par des analyses enzymatiques basées sur la peptidase-1 (Ferrier et al., 1980, 1983 ; Dournon et Houillon, 1984). Ils sont fonctionnels, ce que prouve la présence de spermatophores sur le fond des aquariums d'élevage. II reste à vérifier leur pouvoir fécondant en les croisant avec des femelles standard ZW ou mieux avec des femelles thelygènes WW. Ce dernier cas permet d'envisager l'obtention systématique de Pleurodèles à génotype $W W$ et de phénotype mâle ou femelle selon la température d'élevage.

Disposant de progéniteurs à génotype particulier et orientant à volonté la différenciation des gonades de leurs descendants, il devient possible d'envisager sous des aspects nouveaux le problème fondamental de la différenciation sexuelle. Connaissant en outre, d'une manière plus précise, les stades où interviennent certains événements critiques de la différenciation des glandes génitales, nos recherches peuvent maintenant s'orienter vers des analyses biochimiques et des études expérimentales pour élucider les processus impliqués dans l'organogenèse gonadique des Vertébrés.

Reçu en janvier 1985.

Acceptê en avril 1985.

Remerciements. - Nous remercions le Dr Guillez, le Professeur Cherruault du laboratoire Medimat (Université Pierre-et-Marie-Curie, Paris) et le Dr Pieau de I'Institut Jacques Monod (C.N.R.S.-Université Paris VII) pour leurs précieux conseils. Ce travail a été réalisé avec l'aide financière de la D.G.R.S.T., contrat $n^{\circ}$ 79.7.1221, puis du C.N.R.S., A.T.P. "Biologie du Développement et de la Reproduction ».

\section{Références}

BULL J. J., 1980. Sex determination in reptiles. Quart. Rev. Biol., 55, 3-21.

BULL J. J., 1983. Evolution of sex determining mechanisms. The Benjamin/Cummings Publ. Co. Inc., 109-144, London, Amsterdam, Don Mills (Ontario), Sidney, Tokyo.

BULL J. J., VOGT R. C., 1981. Temperature sensitive periods of sex determination in emydid turtles. J. exp. Zool., 218, 435-440.

DOURNON C., 1981. Action d'une température d'elevage de $30^{\circ} \mathrm{C}$ sur la morphogenèse, l'inversion fonctionnelle du phénotype sexuel et la prolifération des cellules germinales chez l'Amphibien Urodèle Pleurodeles waltiii Michah., Th. Doct. Etat Sci. nat., Univ. P. et M. Curie, Paris, $204 \mathrm{p}$. 
DOURNON C., GUILLET F., BOUCHER D., LACROIX J. C., 1984. Cytogenetic and genetic evidence of male sexual inversion by heat treatment in the newt Pleurodeles poireti. Chromosoma (Berl.), 90, 261-264.

DOURNON C., HOUILLON Ch., 1984. Démonstration génétique de l'inversion fonctionnelle du phénotype sexuel femelle sous l'action de la température d'élevage chez l'Amphibien Urodèle: Pleurodeles waltiii Michah. Reprod. Nutr. Dévelop., 24, 361-378.

FERRIER V., JAYLET A., CAYROL C., GASSER F., BUISAN J. J., 1980. Etude électrophorétique des peptidases érythrocytaires chez Pleurodeles waltiii (Amphibien Urodèle) : mise en évidence d'une liaison avec le sexe. C. R. Acad. Sci. Paris, Sér. D, 290, 571-574.

FERRIER V., GASSER F., JAYLET A., CAYROL C., 1983. A genetic study of various enzyme polymorphisms in Pleurodeles waltiii (urodele amphibian). Il-peptidases : demonstration of sex linkage. Biochem. Genet., 21, 535-549.

GALLIEN L., 1950. Inversion du sexe (féminisation) chez I'Urodèle Pleurodeles waltlii Michah. traité par le benzoate d'œstradiol. C. R. Acad. Sci. Paris, 231, 919-920.

GALLIEN L., 1952. Elevage et comportement du Pleurodèle au laboratoire. Bull. Soc. Zool. Fr., 77, 456-461.

GALLIEN L., DUROCHER M., 1957. Table chronologique du développement chez Pleurodeles waltlii Michah. Bull. Biol., Fr. Belg., 91, 97-114.

HOUILLON Ch., 1956. Recherches expérimentales sur la dissociation médullo-corticale dans l'organogenèse des gonades chez le triton, Pleurodeles waltlii Michah. Bull. Biol. Fr. Belg., 90, 359-445.

HOUILLON Ch., DOURNON C., 1978. Inversion du phénotype sexuel femelle sous l'action d'une température élevée chez l'Amphibien Urodèle, Pleurodeles waltlii Michah. C. R. Acad. Sci. Paris, Sér. D, 286, 1475-1478.

LACROIX J. C., 1968. Variations expérimentales ou spontanées de la morphologie et de l'organisation des chromosomes en écouvillon dans le genre Pleurodeles (Amphibien Urodèle). Ann. Embryol. Morph., 1, 205-248.

LACROIX J. C., 1970. Mise en évidence sur les chromosomes en écouvillon de Pleurodeles poireti Gervais, Amphibien Urodèle, d'une structure liée au sexe, identifiant le bivalent sexuel et marquant le chromosome W. C. R. Acad. Sci. Paris, Sér. D, 271, 102-104.

PIEAU C., 1975. Temperature and sex differentiation in embryos of two chelonians, Emys orbicularis L. and Testudo graeca L., 332-339. In R. REINBOTH Intersexuality in the animal kingdom. Springer Verlag. Berlin, Heidelberg, New York.

PIEAU C., 1985. Déterminisme du sexe chez les Reptiles; influence de facteurs épigénétiques. Bull. Soc. Zool. Fr., (sous presse).

PIEAU C., DORIZZI M., 1981. Determination of temperature sensitive stages for sexual differentiation of the gonads in embryos of the turtle, Emys orbicularis. J. Morphol., 170, 373-382.

YNTEMA C. L., 1979. Temperature levels and periods of sex determination during incubation of eggs of Chelydra serpentina. J. Morph., 159, 17-28.

YNTEMA C. L., MROSOVSKY N., 1982. Critical periods and pivotal temperatures of sexual differentiation in loggerhead sea turtles. Canad. J. Zool., 60, 1012-1016. 